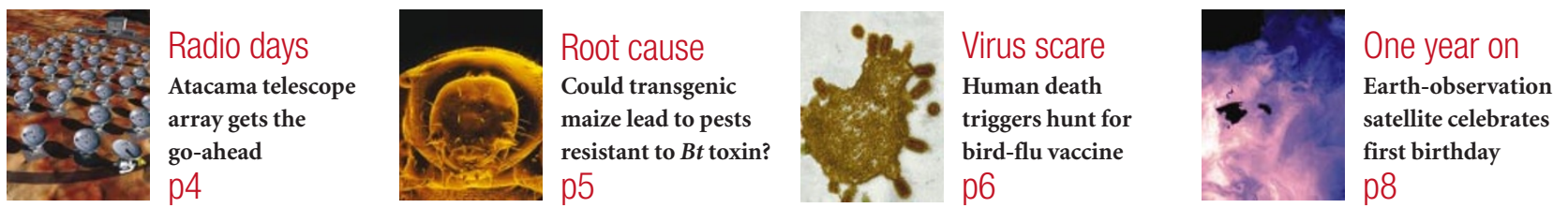

\title{
Astronomers urge NASA not to cut corners on Hubble successor
}

Tony Reichhardt, Washington

Researchers are rallying in defence of a key instrument that NASA is considering dropping from its next space telescope - the project ranked by US astronomers as their highest single priority.

Project scientists and congressional staff confirm that the space agency is considering axeing the Mid-Infrared Instrument (MIRI) - one of three instruments to go on the James Webb Space Telescope (JWST) in a last-ditch effort to keep the project on time and within budget. The JWST, formerly known as the Next Generation Space Telescope, is due to replace the spectacularly successful Hubble Space Telescope in 2011 as the premier observatory in space.

But ditching MIRI would be a "terrible scientific loss", says Christopher McKee, an astrophysicist at the University of California, Berkeley, and co-chair of the National Academy of Sciences panel that gave the telescope top ranking in its 2000 decadal review. Others agree that MIRI should be preserved, even if it means delaying the telescope's launch or cancelling other projects to pay for it.

The JWST has risen steadily in price from its original — and, some would say, unrealistic - target of $\$ 500$ million in the late 1990 s to $\$ 1.6$ billion and going, not counting launch. Last November, NASA asked project managers, scientists and the telescope's builder, Northrop Grumman Space Technology, to cut costs to stay within the budget. But no obvious solutions have surfaced apart from delaying the launch or reducing the telescope's capability. "We still have a problem," says Eric Smith, programme scientist for the JWST at NASA headquarters.

The James Webb telescope, named for the administrator who guided NASA during the Apollo Moon landings, will have a mirror 6 metres in diameter, over twice that of Hubble's mirror. It will observe in the infrared waveband, where it can detect evidence of the oldest stars and galaxies in the Universe. A US-built instrument called NIRCam (NearInfrared Camera) will cover infrared wavelengths from the edge of visible light up to 5 micrometres. MIRI - whose cost is shared by the United States and Europe — would

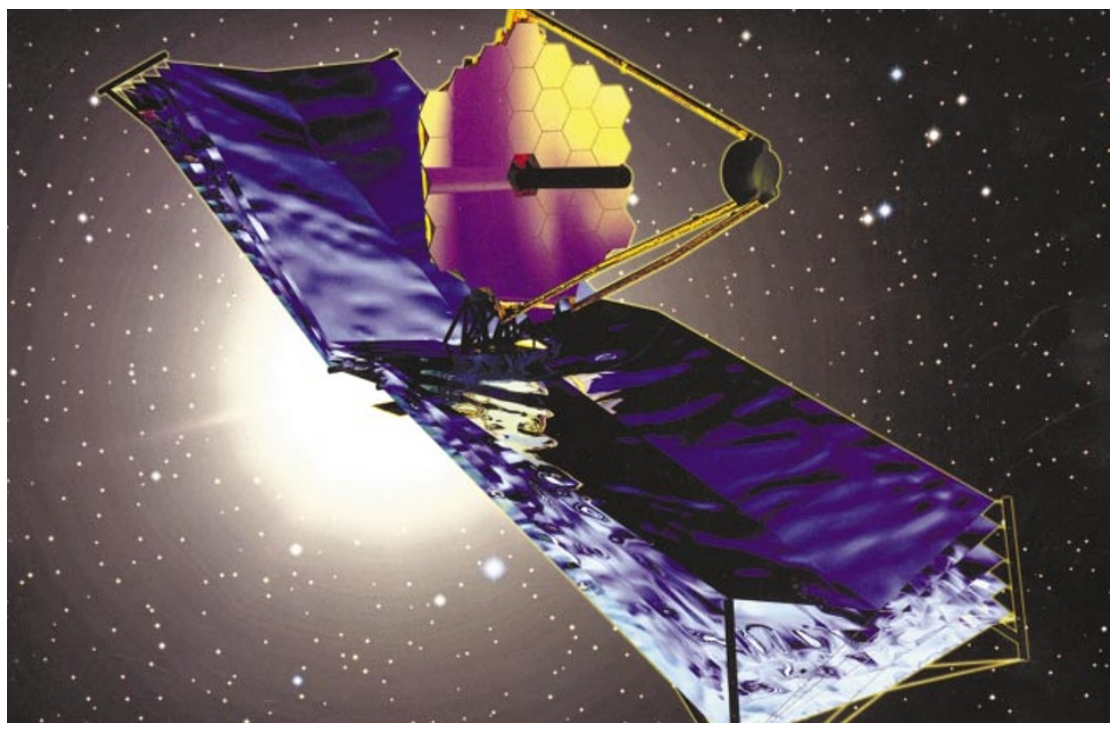

The James Webb Space Telescope could lose one of its three instruments unless more cash is found.

extend the range to 28 micrometres, and a third European instrument called NIRSpec (Near-Infrared Spectrograph) would complement NIRCam by taking spectra of stars and galaxies in the same infrared wavelengths.

Although NIRCam is the telescope's prime instrument, it needs MIRI's corroborating observations to answer some key scientific questions. At longer wavelengths, for example, it will have trouble distinguishing between the Universe's very first stars and those born later. MIRI will also allow studies of objects in the distant Kuiper belt in our own Solar System and of planet-forming disks around other stars.

In a letter sent on 29 January to Anne Kinney, head of NASA's Astronomy and Physics Division, McKee and panel co-chair Joseph Taylor of Princeton University called MIRI "an essential part of the complement of instruments that will enable JWST to have a revolutionary impact on astronomy and astrophysics". They urged NASA to consult with the science community before making any decision, and if necessary, to consider "descoping or delaying other projects in order to ensure the success of JWST".

Ironically, just as rumours were circulat- ing last week that NASA might cut its $\$ 100$ million investment in MIRI, the European Space Agency's Science Programme Committee gave approval for its half of the instrument, and agreed that the agency should provide an Ariane launcher for the telescope. Smith calls the Ariane offer "fantastic news", but the project would still be short of at least $\$ 100$ million. And although Smith says NASA has reached no decision, MIRI is the obvious place to cut if the telescope is to stay on time and on budget.

Last month a NASA advisory subcommittee suggested delaying the launch by up to two years to save MIRI. In a letter sent on 2 February to the head of NASA's science advisory committee, subcommittee chairman Alan Dressler of the Carnegie Observatories wrote that "MIRI is critical for mission success".

NASA is loathe to delay the JWST, as this would increase the cost of the project and give ammunition to critics of its projectmanagement skills. Embarrassed by budget overruns on the International Space Station, the agency badly needs to demonstrate fiscal responsibility. Rather than ask for more money, says Smith, "we're going to solve the problem within the space science office". 